\title{
A New Method to Assess Sinus Rhythm Maintenance Likelihood Before Electrical Cardioversion of Persistent Atrial Fibrillation
}

\author{
R Alcaraz ${ }^{1}$, JJ Rieta $^{2}$ \\ ${ }^{1}$ Innovation in Bioengineering Research Group. University of Castilla-La Mancha, Cuenca, Spain \\ ${ }^{2}$ Biomedical Synergy. Valencia University of Technology, Valencia, Spain
}

\begin{abstract}
For many patients in persistent Atrial Fibrillation $(A F)$, normal sinus rhythm (NSR) restoration use to be required to reduce the risk of stroke and improve cardiac output. Electrical cardioversion (ECV) is the most effective alternative to revert $A F$ to sinus rhythm. However, because of the high risk of $A F$ recurrence (especially during the first 2 weeks following the procedure) and because of potential collateral effects of ECV, it is clinically important to predict NSR maintenance after ECV before it is attempted. Thereby, the aim of this work is to obtain a robust and noninvasive predictor of NSR maintenance after ECV. The organization degree of the atrial activity (AA) obtained from surface electrocardiographic (ECG) recordings was estimated using a non-linear regularity index because it can be hypothesized that $A F$ recurrence can be greater in those patients who present a highly disorganized AA. With the proposed strategy, $90.24 \%$ sensitivity and $78.57 \%$ specificity were obtained. Therefore, therapeutic interventions for the treatment of persistent AF could be improved, since unsuccessful electrical cardioversion and the consequent risks for the AF patients could be avoided.
\end{abstract}

\section{Introduction}

Atrial Fibrillation (AF) is the most commonly sustained cardiac arrhythmia in clinical practice, with a prevalence of $0.5 \%$ in the adult population [1], rising to $10 \%$ or more in those over 75 years $[2,3]$. This disease can be divided into different forms, namely paroxysmal AF (selfterminating within 7 days), persistent $\mathrm{AF}$ (interventions are required for its termination), and permanent AF (sinus rhythm cannot be restored) [2]. For many patients in persistent $\mathrm{AF}$, restoration and maintenance of normal sinus rhythm (NSR) is the main therapeutic goal because symptoms, cardiac output, and exercise tolerance are improved whereas the risk of stroke is reduced [2]. Thus, the first step in the rhythm control strategy is generally cardioversion. While chemical-induced cardioversion is sometimes possible, particularly with amiodarone [4, 5], it is generally more unsuccessful than electrical cardioversion (ECV), specially if the arrhythmia has been present for more than 24 hours [6]. However, because of the high AF risk of recurrence, especially during the first 2 weeks following the procedure [7] and because of the potential secondary effects of ECV $[8,9]$, it would be clinically useful to predict NSR maintenance after electrical cardioversion before it is attempted. In this way, the risks of cardioversion could be avoided for those patients with low NSR maintenance probability, and for the health care provider, the clinical cost could be optimized because unproductive treatment time and bed usage could be reduced.

To date, numerous studies have attempted to find invasive and non-invasive parameters (clinical, electrophysiological, demographic, etc.) for the prediction of electrical cardioversion outcome for AF. However, very different and, consequently, inconclusive results have been obtained [10]. Thereby, the aim of this work is to obtain a new robust and non-invasive predictor of NSR maintenance after ECV before it is attempted. In this respect, the atrial activity (AA) organization was estimated noninvasively, because it can be hypothesized that NSR maintenance would be more likelihood in patients who present a highly organized AA. This hypothesis is based on the observation that the more disorganized the AA, the higher the number of propagating wavelets [11], and the larger the atrial volume that could support reentries propagation after the shock [12].

In order to estimate the AA organization obtained from surface electrocardiographic (ECG) recordings, Sample Entropy (SampEn), which is a non-linear index for quantifying time series regularity [13], was selected because nonlinearity, as necessary condition for a chaotic behavior, is present in the diseased heart with AF at cellular level, and the electrical remodeling in AF is a far-from-linear process [14]. This phenomenon can be described as the progressive shortening of effective atrial refractory periods, thus increasing the number of simultaneous reentries and, as a consequence, the perpetuation of AF [2]. Moreover, 
in previous works it has been shown that Sample Entropy is a robust organization estimator of the AA obtained from surface ECG recordings [15, 16].

\section{Materials}

Forty patients (15 men and 25 women) with persistent AF lasting more than 30 days, undergoing the first attempt of electrical cardioversion were followed during four weeks. A standard 12-lead ECG was acquired prior to cardioversion. All signals were digitized at a sampling rate of $1024 \mathrm{~Hz}$ and 16-bit resolution by means of Cardiolab System in the electrophysiology laboratory during ECV protocol. In order to process these signals, a 30 seconds-length AF segment preceding the ECV was extracted for each patient. After the ECV, NSR was not restored in 5 patients $(12.5 \%)$ whereas in $21(52.5 \%)$ NSR duration was below one month. In the remaining 14 patients (35\%) NSR was maintained. All patients were in drug treatment with amiodarone.

The ECG recordings were preprocessed in order to reduce noise, nuisance interferences and improve later analysis. Firstly, baseline wander was removed making use of bidirectional high pass filtering with $0.5 \mathrm{~Hz}$ cutt-off frequency [17]. Secondly, high frequency noise was reduced with an eight order bidirectional IIR Chebyshev low pass filtering, whose cut-off frequency was $70 \mathrm{~Hz}$ [18]. Finally, powerline interference was removed through adaptive notch filtering, which preserves the ECG spectral information [19].

\section{Methods}

\subsection{Strategy to predict NSR maintenance}

The analysis of the AA obtained from the surface ECG is complicated by the simultaneous presence of ventricular activity, which is of much greater amplitude. Whereby, the AA signal of lead $V_{1}$ was firstly extracted using the average QRST template cancellation method [20]. Previous works have shown that AF is dominant in this lead, whereby it was chosen for the analysis [21].

Bearing in mind that the median beat cannot represent each individual beat accurately, since QRST morphology is affected by respiration, patient movement, etc., QRST residuals and noise are often present in the remainder ECG [21]. These nuisance signals can degrade AA organization estimation using non-linear regularity indexes, which could provoke unsuccessful results. Hence, in order to reduce the presence of noise, ventricular residues and any other nuisance signal in the AA, a wavelet decomposition analysis of AA is proposed. Seven levels wavelet decomposition was applied to AA signals because the seventh discrete scale (sub-band corresponding to $4-8 \mathrm{~Hz}$ ) covers most typical AA frequency range, that is around $3-9 \mathrm{~Hz}$ [22]. This scale was reconstructed back to the timedomain and its organization was estimated making use of Sample Entropy (SampEn), which is notably independent on record length and obtains coherent results when short and noisy data sets are analyzed [13]. A more detailed mathematic description of SampEn can be found in [13].

Because of there are not established rules for the choice of a wavelet family, several orthogonal families were tested. The best results were obtained with Biorthogonal wavelet family (order 4.4). A wide description of Wavelet Transform and wavelet families can be found in [23].

\subsection{Statistical analysis}

Results were expressed as mean \pm standard deviation, unless otherwise specified. In order to evaluate the organization analysis predictive cability for the NSR maintenance, receiver operating characteristic (ROC) curves were constructed. Different thresholds or cutoff points (SampEn values) were selected and the sensitivity/specificity pair for each one of them was calculated. Sensitivity (the true positive rate) is the ECVs relapsing to AF proportion correctly classified (SampEn value higher than the cutoff point), whereas specificity (the true negative rate) represents the ECVs resulting in NSR percentage correctly recognized (SampEn value lower than the cutoff point). The closest point to $100 \%$ sensitivity and specificity was selected as optimum SampEn threshold. The $t$-student test was used to determine whether there was any significant difference between the groups. A two-tailed value of $p<0.05$ was considered statistically significant.

\section{Results}

With the proposed methodology, $90.47 \%$ sensitivity and $78.57 \%$ specificity were obtained, see Fig. 1(a). The ROC curve provided 0.0990 as optimum SampEn discrimination threshold between recordings relapsing to AF and resulting in NSR after one month. Fig. 1(b) shows the SampEn values for the all analyzed signals together with the mean and standard deviation values for each group. Note that the patients who resulted in NSR presented lower Sam$\mathrm{pEn}$ values $(0.0930 \pm 0.0150)$ than those who relapsed to $\mathrm{AF}(0.1136 \pm 0.0151)$. Indeed, both groups were statistically distinguishable, given that statistic significance was lower than 0.0001. In addition, the five patients who relapsed to AF immediately after the ECV were also studied separately and they presented the lowest AA organization $(0.1255 \pm 0.0165)$, thus reinforcing the robustness of the presented approach. 


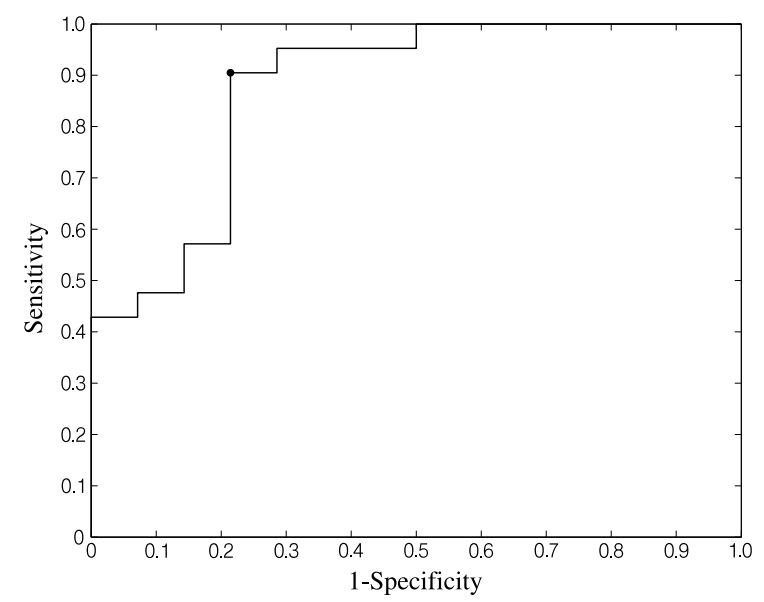

(a)

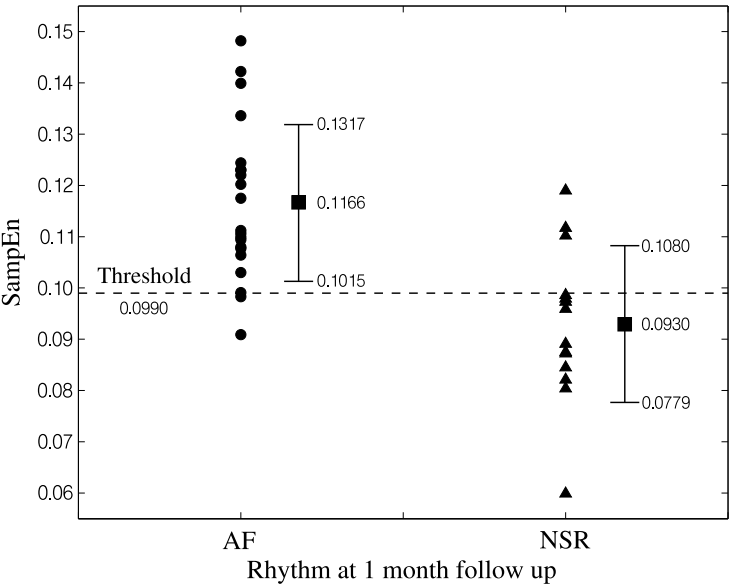

(b)

Figure 1. (a) Receiver Operating Characteristic (ROC) curve calculated with the obtained organization values. (b) Classification into ECVs resulting in NSR and relapsing to AF after 4 weeks following ECV.

\section{Discussion and conclusions}

The organization analysis results show that recordings relapsing to AF present higher AA disorganization than those resulting in NSR after 4 weeks. This observation agrees with findings reported in other works, such as: (i) the higher the AA organization, the higher the success rates in $\mathrm{AF}$ cardioversion [24, 11], (ii) the higher the level of AA organization, the lower the energy required for successful cardioversion [12] and (iii) paroxysmal AF requires less energy for cardioversion than persistent AF [25]. These previous observations highlight the fact that, when a higher number of reentries are wandering throughout the atrial tissue, a lower probability of successful electrical cardioversion is obtained. One possible explanation could be that a low degree of AA organization might result in an increased mass of atrial myocardium that is not fully excitable [24]. Moreover, the necessity of a lower refractory period prolongation into a lower volume of atrial tissue, when the AA is highly organized, could also justify the obtained results.

The five patients in which NSR was not immediately restored after ECV presented the lowest AA organization. This observation also agrees with the outcomes reported with invasive recordings $[24,11]$ and, thereby, the obtained results consistency is increased.

Other authors have also tried to estimate AF organization for predicting NSR maintenance after ECV. Holmqvist et. al. [26] evaluated a parameter obtained from timefrequency analysis of the atrial signals, such as harmonic decay [22]. This parameter was designed to be an index of the waveform shape (and indirectly organization) of the atrial component of the ECG, but a low number of recordings relapsing to AF were correctly identified (47\%). In [27], ventricular rhythm was analyzed using three-dimensional RR intervals plots, quantifying clustering of RR intervals. The authors speculated that RR intervals clustering represents a relatively high organization degree of atrial fibrillatory activity, and hypothesized that ECV would be more effective in patients with clustering. However, only $53.8 \%$ of patients who relapsed to AF (during the first 4 hours following ECV) were correctly discerned. These results also motivated the use of non-linear regularity indexes for estimating the AA organization.

Hence, the obtained results presented in this work prove that the noninvasive and non-linear assessment of AA organization is a reliable and robust method to determine the individual risk for early AF relapse, thereby avoiding unnecessary cardioversion attempts.

\section{Acknowledgements}

This work was partly supported by the projects 20070086 from the R+D+i Vice-rectorate of the Valencia University of Technology, GV06/299 from Consellería de Empresa, Universidad y Ciencia de la Generalitat Valenciana and TEC2007-64884 from the Spanish Ministry of Education and Science.

\section{References}

[1] Kannel WB, Abbott RD, Savage DD, McNamara PM. Epidemiologic features of chronic atrial fibrillation: the Framingham study. N Engl J Med Apr 1982;306(17):1018-1022.

[2] Fuster V, Rydén LE, Cannom DS, Crijns HJ, Curtis AB, Ellenbogen KA, et. al. ACC/AHA/ESC 2006 guidelines for the management of patients with atrial fibrillation: a report of the American College of Cardiology/American Heart Association task force on practice guidelines and the european society of cardiology committee for practice guide- 
lines (writing committee to revise the 2001 guidelines for the management of patients with atrial fibrillation): developed in collaboration with the european heart rhythm association and the heart rhythm society. Circulation Aug 2006; 114(7):e257-e354.

[3] Furberg CD, Psaty BM, Manolio TA, Gardin JM, Smith VE, Rautaharju PM. Prevalence of atrial fibrillation in elderly subjects (the Cardiovascular Health Study). Am J Cardiol Aug 1994;74(3):236-241.

[4] McNamara RL, Tamariz LJ, Segal JB, Bass EB. Management of atrial fibrillation: review of the evidence for the role of pharmacologic therapy, electrical cardioversion, and echocardiography. Ann Intern Med Dec 2003; 139(12):1018-1033.

[5] Singh BN, Singh SN, Reda DJ, Tang XC, Lopez B, Harris CL, Fletcher RD, Sharma SC, Atwood JE, Jacobson AK, Lewis HD, Raisch DW, Ezekowitz MD, Investigators SAAFETST. Amiodarone versus sotalol for atrial fibrillation. N Engl J Med May 2005;352(18):1861-1872.

[6] Gall NP, Murgatroyd FD. Electrical cardioversion for AFthe state of the art. Pacing Clin Electrophysiol Apr 2007; 30(4):554-567.

[7] Tieleman RG, Gelder ICV, Crijns HJ, Kam PJD, Berg MPVD, Haaksma J, Woude HJVD, Allessie MA. Early recurrences of atrial fibrillation after electrical cardioversion: a result of fibrillation-induced electrical remodeling of the atria? J Am Coll Cardiol Jan 1998;31(1):167-173.

[8] Ebrahimi R, Rubin SA. Electrical cardioversion resulting in death from synchronization failure. Am J Cardiol Jul 1994; 74(1):100-102.

[9] Gale DW, Grissom TE, Mirenda JV. Titration of intravenous anesthetics for cardioversion: a comparison of propofol, methohexital, and midazolam. Crit Care Med Oct 1993; 21(10):1509-1513.

[10] Holmqvist F, Stridh M, Waktare JEP, Sörnmo L, Olsson SB, Meurling CJ. Atrial fibrillatory rate and sinus rhythm maintenance in patients undergoing cardioversion of persistent atrial fibrillation. Eur Heart J Sep 2006;27(18):2201-2207.

[11] Sih HJ, Zipes DP, Berbari EJ, Olgin JE. A high-temporal resolution algorithm for quantifying organization during atrial fibrillation. IEEE Trans Biomed Eng Apr 1999; 46(4):440-450.

[12] Calcagnini G, Censi F, Michelucci A, Bartolini P. Descriptors of wavefront propagation. Endocardial mapping of atrial fibrillation with basket catheter. IEEE Eng Med Biol Mag 2006;25(6):71-78.

[13] Richman JS, Moorman JR. Physiological time-series analysis using approximate entropy and sample entropy. Am J Physiol Heart Circ Physiol Jun 2000;278(6):H2039H2049.

[14] Bollmann A. Quantification of electrical remodeling in human atrial fibrillation. Cardiovasc Res 2000;47:207-209.

[15] Alcaraz R, Rieta JJ. Non-linear organization analysis of paroxysmal atrial fibrillation. 29th Annual International
Conference of the IEEE Engineering in Medicine and Biology Society 2007; in press.

[16] Alcaraz R, Rieta JJ. Wavelet bidomain regularity analysis to predict spontaneous termination of atrial fibrillation. 29th Annual International Conference of the IEEE Engineering in Medicine and Biology Society 2007; in press.

[17] Dotsinsky I, Stoyanov T. Optimization of bi-directional digital filtering for drift suppression in electrocardiogram signals. J Med Eng Technol 2004;28(4):178-180.

[18] Sun Y, Chan K, Krishnan SM. ECG signal conditioning by morphological filtering. Comput Biol Med Nov 2002; 32(6):465-479.

[19] Ferdjallah M, Barr RE. Adaptive digital notch filter design on the unit circle for the removal of powerline noise from biomedical signals. IEEE Trans Biomed Eng Jun 1994; 41(6):529-536.

[20] Slocum J, Sahakian A, Swiryn S. Diagnosis of atrial fibrillation from surface electrocardiograms based on computerdetected atrial activity. J Electrocardiol Jan 1992;25(1):1-8.

[21] Petrutiu S, Ng J, Nijm GM, Al-Angari H, Swiryn S, Sahakian AV. Atrial fibrillation and waveform characterization. A time domain perspective in the surface ECG. IEEE Eng Med Biol Mag 2006;25(6):24-30.

[22] Stridh M, Sörnmo L, Meurling CJ, Olsson SB. Sequential characterization of atrial tachyarrhythmias based on ECG time-frequency analysis. IEEE Trans Biomed Eng Jan 2004;51(1):100-114.

[23] Mallat S. A Wavelet Tour of Signal Processing. Academic Press, 1999. ISBN 0-12-466606-X.

[24] Everett TH, Kok LC, Vaughn RH, Moorman JR, Haines DE. Frequency domain algorithm for quantifying atrial fibrillation organization to increase defibrillation efficacy. IEEE Trans Biomed Eng Sep 2001;48(9):969-978.

[25] Lau CP, Lok NS. A comparison of transvenous atrial defibrillation of acute and chronic atrial fibrillation and the effect of intravenous sotalol on human atrial defibrillation threshold. Pacing Clin Electrophysiol Oct 1997;20(10 Pt 1):2442-2452.

[26] Holmqvist F, Stridh M, Waktare JEP, Roijer A, Sörnmo L, Platonov PG, Meurling CJ. Atrial fibrillation signal organization predicts sinus rhythm maintenance in patients undergoing cardioversion of atrial fibrillation. Europace Aug 2006;8(8):559-565.

[27] Berg MPVD, Noord TV, Brouwer J, Haaksma J, Veldhuisen DJV, Crijns HJGM, Gelder ICV. Clustering of RR intervals predicts effective electrical cardioversion for atrial fibrillation. J Cardiovasc Electrophysiol Sep 2004;15(9):10271033.

Address for correspondence:

Raúl Alcaraz Martínez

E. U. Politécnica de Cuenca

Campus Universitario

16071 Cuenca (Spain)

raul.alcaraz@uclm.es 